\title{
A Markov Model of the Cost-Effectiveness of Pharmacist Care for Diabetes in Prevention of Cardiovascular Diseases: Evidence from Kaiser Permanente Northern California
}

\author{
Junhua Yu, PhD, MS; Bijal M. Shah, PhD; Eric J. Ip, PharmD, BCPS, CSCS, CDE; \\ and James Chan, PharmD, PhD
}

\begin{abstract}
BACKGROUND: It has been demonstrated in previous studies that pharmacist management of patients with type 2 diabetes mellitus (T2DM) in the outpatient setting not only improves diabetes-related clinical outcomes such as hemoglobin A1c but also blood pressure (BP), total cholesterol (TC), and quality of life. Improved control of BP and TC has been shown to reduce the risks of cardiovascular disease (CVD), which has placed a heavy economic burden on the health care system. However, no study has evaluated the cost-effectiveness of pharmacist intervention programs with respect to the long-term preventive effects on CVD outcomes among T2DM patients.
\end{abstract}

OBJECTIVES: To (a) quantify the long-term preventive effects of pharmacist intervention on CVD outcomes among T2DM patients using evidence from a matched cohort study in the outpatient primary care setting and (b) assess the relative cost-effectiveness of adding a clinical pharmacist to the primary care team for the management of patients with T2DM based on improvement in CVD risks with the aid of an economic model.

METHODS: Clinical data between the periods of June 2007 to February 2010 were collected from electronic medical records at 2 separate clinics at Kaiser Permanente (KP) Northern California, 1 with primary care physicians only (control group) and the other with the addition of a pharmacist (enhanced care group). Patients in the enhanced care group were matched 1:1 with patients in the control group according to baseline characteristics that included age, gender, A1c, and Charlson comorbidity score. The estimated 10-year CVD risk for both groups was calculated by the United Kingdom Prospective Diabetes Study (UKPDS) Risk Engine (version 2) based on age, sex, race, smoking status, atrial fibrillation, duration of diabetes, levels of A1c, systolic BP (SBP) and TC, and high-density lipoprotein cholesterol (HDL-C)observed at 12 months. There was no statistical difference in the baseline clinical inputs to the Risk Engine (A1c $[P=0.115]$, SBP $[P=0.184]$, TC $[P=0.055]$, and HDL-C $[P=0.475])$ between the 2 groups. A Markov model was developed to simulate the estimated CVD outcomes over 10 years and to estimate cost-effectiveness. The final outcomes examined included incremental cost and effectiveness measured by life years and per quality-adjusted life year gained. Both deterministic sensitivity analysis (SA) and probabilistic SA were conducted to examine the robustness of the results.

RESULTS: The estimated risks for coronary heart disease (CHD) and stroke (both nonfatal and fatal) at the end of the follow-up were consistently lower in the enhanced care group compared with the control group, even though baseline risks in both groups were similar. The absolute risk reduction (ARR) between the enhanced care and control groups increased over time. For example, the ARR for nonfatal CHD risk in year 1 was $0.5 \%(1.2 \%$ vs. $0.7 \%$ ), whereas the ARR increased to $5.5 \%$ in year 10 (14.8\% vs. $9.3 \%)$. Similarly, the ARR between the enhanced care and the control groups was calculated as $0.3 \%$ for fatal CHD in year 1 and increased to $4.6 \%$ in year 10 . Results from the Markov model suggest that the enhanced care group was shown to be a dominant strategy (less expensive and more effective) compared with the control group in the 10-year evaluation period in the base-case (average or mean results) scenario. Sensitivity analysis that took into account the uncertainty in all important variables, such as wage of pharmacists, utility weight (the degree of preference individuals have for a particular health state or condition), response rate to pharmacists' care, and uncertainty associated with the estimated 10 years of CVD risk, revealed that the relative value of enhanced care was robust to most of the variations in these parameters. Notably, the level of cost-effectiveness measured by net monetary value depends on the time horizon adopted by the payers and the magnitude of CVD risk reduction. The enhanced care group has a higher chance of being considered as a cost-effective strategy when a longer time horizon such as a minimum of 4 to 5 years is adopted.

CONCLUSIONS: Adding pharmacists to the health care management team for diabetic patients improves the long-term CVD risks. The longer-term CVD risk reductions were shown to be more dramatic than the short-term reduction. A longer time horizon adopted by health plans in managing T2DM patients has a higher probability of making the intervention cost-effective.

J Manag Care Pharm. 2013;19(2):102-14

Copyright $\odot 2013$, Academy of Managed Care Pharmacy. All rights reserved.

\section{What is already known about this subject}

- The economic burden associated with cardiovascular disease (CVD) complications among type 2 diabetes mellitus (T2DM) patients is substantial. Adults with diabetes are 2-4 times more likely to have heart disease or stroke, and the total direct medical care costs for treating 1 typical established CVD patient can be $\$ 18,953$ per year.

- Pharmacist management of patients with T2DM in the outpatient setting not only improves diabetes-related clinical outcomes such as hemoglobin Alc but also the secondary outcomes such as blood pressure (BP) and cholesterol levels, which are highly predictive of CVD risks.

- Pharmacist disease management programs have been shown to be cost-effective when considering labor and program costs. However, no studies have evaluated the cost-effectiveness of pharmacist intervention programs with respect to the long-term CVD outcomes among T2DM patients using a rigorous modeling approach. 


\section{A Markov Model of the Cost-Effectiveness of Pharmacist Care for Diabetes in Prevention of Cardiovascular Diseases: Evidence from Kaiser Permanente Northern California}

\section{What this study adds}

- The estimated long-term risks for coronary heart disease (CHD) and stroke (both nonfatal and fatal) at the end of follow-up were consistently lower in the enhanced care group compared with the control group, even though baseline risks in both groups were similar.

- The absolute risk reduction (ARR) between the enhanced care and control groups increased over time. For example, the ARR in the nonfatal CHD risk in year 1 was $0.5 \%$ (1.2\% vs. $0.7 \%$, whereas the ARR increased to $5.5 \%$ in year 10 (14.8\% vs. 9.3\%). Similarly, the ARR between the enhanced care and the control groups was calculated as $0.3 \%$ for fatal CHD in year 1 and increased to $4.6 \%$ in year 10 .

- The Markov model constructed using data from a matched cohort study demonstrated that the enhanced care group dominated the control group with lower treatment cost $(\$ 35,740$ vs. $\$ 44,528)$ per patient and more life years (8.9 vs. 8.1 years) and qualityadjusted life-years (QALY; 5.51 vs. 5.02 years) over the 10-year period.

- The results were robust to almost all possible variations of the relevant parameters in the model except for the time horizon of the health plan. Based on our research, if health plans were willing to pay $\$ 50,000 / \mathrm{QALY}$, it would take at least 4-5 years for the addition of clinical pharmacists to the health care team to be cost-effective without any uncertainty.

$\mathrm{T}$ ype 2 diabetes mellitus (T2DM) is an independent risk factor for cardiovascular disease (CVD) and is increasingly being recognized as a controllable risk factor. Adults with diabetes are 2-4 times more likely to have a heart disease or a stroke, which is the cause of death in at least $65 \%$ of these patients. ${ }^{1}$ The total direct medical care costs for treating an established CVD patient can be $\$ 18,953$ per year. ${ }^{2}$ In particular, the costs for patients who experience a secondary CVD hospitalization are 4.5 times higher compared with those who avoid subsequent inpatient stays. ${ }^{2}$ Thus, a large proportion of the economic burden of T2DM can be attributed to cardiovascular complications, and substantial cost savings can be achieved through effective prevention of CVD.

Pharmacist care for T2DM patients can be instrumental in the prevention of CVD. It has been demonstrated in numerous studies that pharmacist management of patients with T2DM in the outpatient setting can improve glycemic levels such as hemoglobin $\mathrm{Alc}^{3}$ along with blood pressure (BP), cholesterol levels, ${ }^{4,5}$ and overall quality of life (QOL). ${ }^{3,4,6}$ Since BP and cholesterol levels are significant predictors of long-term macrovascular disease such as coronary artery disease, stroke, and peripheral vascular disease, ${ }^{7}$ better control of these clinical markers would presumably decrease CVD risk among diabetic patients. The intervention we attempted to evaluate in this study was conducted in Kaiser Permanente (KP) Northern California A team of 16 primary care physicians (PCPs) in the Internal Medicine Department referred their diabetic patients with poor glycemic control (i.e., Alc $>7 \%$ ) to the clinical pharmacist for more stringent control and medical follow-up. The pharmacist managing patients in this study was clinically trained, having completed a Doctor of Pharmacy (PharmD) degree and a 1-year post-doctoral pharmacy residency and having earned a Certified Diabetes Educator credential. The pharmacist prescribed and adjusted medications, ordered laboratory work, ordered and administered immunizations, provided diabetes self-management education, and worked to optimize overall glycemic and cardiovascular care of patients. The study found that in the enhanced care group the mean Alc decreased from 9.5\% to $6.9 \%$ compared with $9.3 \%$ to $8.4 \%$ in the control group $(P<0.001)$ after 12 months. ${ }^{8}$ Compared with the control group, the enhanced care group increased the probability of achieving an $\mathrm{Alc}<7 \%$ (odds ratio (OR) 3.9, $\mathrm{P}<0.001$ ), low-density lipoprotein cholesterol (LDL-C) $<100$ milligrams per deciliter (mg/dL; OR 2.0, $P=0.0152$ ), BP < 130/80 millimeter of mercury (mmHg; OR 2.0, $P=0.0016$ ), and all 3 goals simultaneously (OR 3.2, $P=0.0004)$. The 10 -year coronary heart disease (CHD) risk decreased from $16.4 \%$ to $9.3 \%$ in the enhanced care group compared with $17.4 \%$ to $14.8 \%$ in the control group $(P<0.001){ }^{8}$

Given the clinical benefits of pharmacist intervention programs for diabetic patients, it is important to quantify their economic value in controlling long-term CVD risk factors from the payer perspective. Two studies have explored the economic impact of pharmacist interventions in managing diabetic patients. However, further data are needed to expand the literature to document the preventive outcomes of CVD risks and address methodological issues posed by the existing literature.

The Asheville Project was 1 of the first large-scale studies to document the positive impact of a pharmacist-run diabetes management program on clinical and economic outcomes in the community pharmacy setting. ${ }^{9}$ More recently, the Diabetes Ten City Challenge reported similar results. ${ }^{10}$ Although both studies attempted to assess the economic outcomes of pharmacist intervention, they relied on evidence collected from community pharmacy settings in geographically distinct locations. Furthermore, both studies utilized outcomes measured using a pre- /post-comparison study design without using a concurrent control group. This study design has 2 main disadvantages: inability to control for confounding factors changing over the follow-up period and regression to the mean-both of which may bias the study outcomes. In addition, of the 2 economic studies available (with a control group), only 1 evaluated pharmacist care in the outpatient setting; however, it was limited by documenting only program characteristics and labor costs. ${ }^{11,12}$ In summary, previous economic evaluations have the drawbacks of either relying on evidence lacking generalizability or 


\section{A Markov Model of the Cost-Effectiveness of Pharmacist Care for Diabetes in Prevention of Cardiovascular Diseases: Evidence from Kaiser Permanente Northern California}

\section{FIGURE 1 Markov Model for CVD Events for Patients with Type 2 Diabetes}

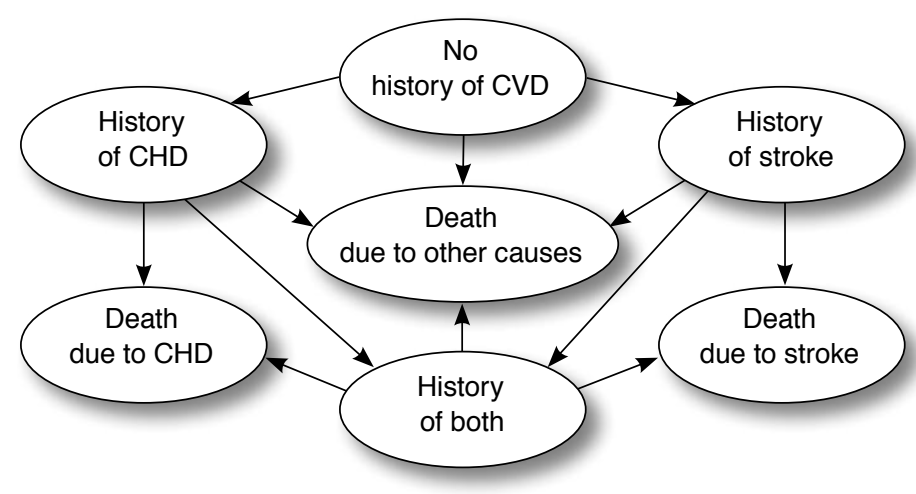

$C H D=$ coronary heart disease; $C V D=$ cardiovascular disease.

omitting economic costs relevant to payers. Most importantly, none of these studies evaluated the cost-effectiveness of pharmacist intervention programs with respect to the long-term CVD outcomes among T2DM patients using a rigorous modeling approach. The need for data on long-term effectiveness of treatment strategies among diabetic patients has long been recognized. ${ }^{13}$

Therefore, the overall goals of this study are to (a) quantify the long-term preventive effects of pharmacist intervention on CVD outcomes among T2DM patients using evidence from a matched cohort study in the outpatient primary care setting and (b) assess the relative cost-effectiveness of adding a clinical pharmacist to the primary care team for the management of patients with T2DM based on improvement in estimated CVD risks with the aid of an economic model.

\section{Methods}

\section{Model Overview}

A Markov model with 1-year cycles was developed to simulate CVD events and death risk for 2 hypothetical cohorts of patients: (1) the enhanced care group where patients were managed by a clinical pharmacist who was integrated as part of the primary care team as a provider and (2) the control group where patients received only the usual care of primary care physicians (PCPs). Figure 1 depicts a simplified presentation of the model. ${ }^{14}$ Patients in both arms entered the model through the "well" state. As patients aged during the 10-year simulation, they could remain free of events, develop a first CHD or stroke, develop recurrent CHD or stroke (either fatal or nonfatal), or die from the events or other natural causes in any model cycle. We assumed that patients who had experienced a first CHD event or stroke would continue to be exposed to the risk for subsequent strokes or CHD events, with a maximum of 3 nonfatal events ( 1 stroke and 2 CHD, 2 strokes and 1 CHD, 3
CHD or 3 strokes). ${ }^{14}$ This model was a Markov model with 11 mutually exclusive health states to take into account various combinations of a maximum total of 3 strokes and CHD events (well, free of history of events, survival from primary stroke, survival from second stroke, survival from third stroke, survival from primary $\mathrm{CHD}$, survival from second $\mathrm{CHD}$, survival from third $\mathrm{CHD}$, survival from CHD once and stroke once, survival from CHD twice [once] and stroke once [twice], event death, and other death). Based on the total time spent in the different health states, the model estimated the expected survival for patients in each treatment arm, and it was combined with cost and quality of life (QOL) data to estimate the relative cost-effectiveness. It is worth noting that stroke and CHD had differing impacts on the utility and costs accumulated over the time horizon of the model. The utility reduction for stroke was different than that for CHD; in addition, the treatment costs for $\mathrm{CHD}$ and stroke also varied. Therefore, it was important to have different health states to identify the exact type of events and the number of events from the point of view of calculation. For example, once patients entered the health state of survival of primary nonfatal CHD, there were 6 possibilities of transitions in the next cycle: (1) transit to the state of survival from CHD once and stroke once if a nonfatal stroke happened; (2) transit to event death if a fatal stroke happened; (3) transit to survival from second CHD if a nonfatal CHD happened; (4) transit to survival of event death if a fatal CHD happened; (5) remain in the state of survival from primary CHD if no event happened; and (6) other nonevent death. CVD risks used in the Markov model were estimated using United Kingdom Prospective Diabetes Study (UKPDS) Risk Engine (version 2) ) $^{8,15}$ with clinical outcomes measured in the matched-cohort study as the inputs. ${ }^{8}$ Specifically, it was developed based on longitudinal data and took into account factors such as age, sex, race, smoking status, atrial fibrillation, duration of diabetes, levels of Alc, systolic BP (SBP), total cholesterol (TC) and high-density lipoprotein cholesterol (HDL-C) after diagnosis of T2DM. ${ }^{15,16}$ The inputs for the Risk Engine are discussed in the following sections (Note: the clinical values used as model inputs in the UKPDS Risk Engine are part of the findings of another study by Ip et $a^{8} .^{8}$.

\section{Model Inputs}

Clinical inputs for CVD risk prediction. Clinical inputs used for the estimation of CVD risks were collected from a retrospective matched-cohort study that took place in 2 clinics based out of KP Northern California, a large health maintenance organization (HMO) with an integrated health care model. KP maintains comprehensive medical utilization data that includes an electronic medical record for all patient encounters, laboratory results, and prescriptions using standardized methods.

The sample selection methods for this study are described elsewhere. ${ }^{8}$ Briefly, data for individual patients were collected concurrently from 2 medical facilities for the purpose of 


\section{A Markov Model of the Cost-Effectiveness of Pharmacist Care for Diabetes in Prevention of Cardiovascular Diseases: Evidence from Kaiser Permanente Northern California}

comparison: the enhanced care group (204 patients) and the control group (407 patients). The 2-site design was utilized to address concerns that chronic disease state management studies may be susceptible to contamination, since pharmacists could have been involved in caring for the control group patients. $^{3}$ Also, the 2-site design eliminated the potential for a "learning effect" at the same site where physicians may "learn" from the recommendations made by the pharmacist and to pass these recommendations on to patients not being seen by the pharmacist. ${ }^{8}$ To further minimize selection bias and ensure patients between the 2 groups were comparable in baseline CVD risks, patients from the 2 groups were matched based on the following characteristics at baseline: age (within 2 years difference), gender, Alc (within 0.8 difference), and Charlson Comorbidity Index (CCI; within 4-point difference). We calculated CCI based on the following baseline characteristics in both groups: CVD with mild or no residual or mini stroke, CVD/stroke/myocardial infarction, dementia, chronic pulmonary disease, ulcer, mild liver disease, hemiplegia, tumor, metastatic solid tumor, leukemia, lymphoma, human immunodeficiency virus but no acquired immunodeficiency syndrome (AIDS), and AIDS. This resulted in 147 matched patients in each group, respectively, in the final analysis. ${ }^{8}$ The primary clinical inputs in the Risk Engine, including Alc, SBP, TC, and HDL-C obtained at the end of the 12-month follow-up for both groups, were used to estimate the 10-year CVD risk. As shown in Part A of Table 1, there were no significant differences in the baseline values of the following variables, which were the primary clinical inputs for the UKPDS Risk Engine: Alc $(P=0.115)$, SBP $(P=0.184)$, TC $(P=0.055)$, and HDL-C $(P=0.475) .{ }^{8}$ However, patients in the enhanced care group had significant improvements relative to patients in the control group on Alc $(P<0.001)$, SBP $(P<0.001)$, and TC $(P<0.001)$ at the end of the 12-month follow-up. No differences were seen for HDL-C $(P=0.2835){ }^{8}$

Transition probabilities. Each arrow in Figure 1 indicates a transition from one health state to another, which occured at yearly intervals. We used the UKPDS Risk Engine to estimate the transition probabilities of various CVD events: absolute risk of fatal and nonfatal CHD and stroke. With the prediction algorithm in this Risk Engine, the observed effects of Alc, SBP, TC, and HDL-C, along with other characteristics at the individual patient level (Part A in Table 1), were translated to the expected 10-year risks for CHD events and stroke (Part B in Table 1). Part B in Table 1 reports the mean risks for the 147 patients in both groups. As shown in Part A Table 1, there was no significant difference between the 2 groups in the 4 major clinical inputs at baseline. Therefore, it is not surprising that there were no significant differences between the 2 groups in the predicted 10-year CVD risk at baseline as a result of matching $(P=0.18$ for $\mathrm{CHD}$ and fatal stroke; $P=0.176$ for $\mathrm{CHD}$;
$P=0.243$ for stroke). ${ }^{8}$ Other transition probabilities include age-dependent noncardiovascular mortality rates, which were derived from the U.S. Vital Statistics. ${ }^{17}$ It is also assumed that a nonfatal stroke or CHD event would increase mortality, with the increased risk measured by the relative risk (RR) of death after the event. According to the literature, the RR for death after stroke and CHD is estimated to be 2.318 and 3.719 (Table 2), respectively.

Part B in Table 1 displays the CVD risk estimated by the UKPDS Risk Engine based on the data collected at the 12-month follow-up. These estimates were used as transition probabilities for the 2 hypothetical cohorts of 55-year-old diabetic patients in the Markov model. After 12 months of followup, the estimated risks for CHD and stroke (both nonfatal and fatal) were consistently lower in the enhanced care group compared with the control group. The yearly risk increased as the patients aged over the 10-year period in each group. However, it is worth noting that absolute risk reduction (ARR) between the enhanced care group and control group increased over time. For example, the ARR in the nonfatal CHD risk in year 1 was $0.5 \%$ ( $1.2 \%$ vs. $0.7 \%$ ), whereas the ARR increased to $5.5 \%$ in year 10 ( $14.8 \%$ vs. 9.3\%) in favor of the enhanced care group. Similarly, the ARR between the enhanced control group and care group was calculated as $0.3 \%$ for fatal CHD in year 1 and increased to $4.6 \%$ in year 10 .

Cost inputs and utilization of medical resources. We conducted the analysis from the perspective of a third-party payer. Assumptions about the cost of CHD and stroke were derived from the literature as shown in Table 3. We used the Medical Consumer Price Index to generate an inflation factor, which was applied to the past cost figures to convert it to 2011 U.S. dollars. A 3\% discount rate was applied to all costs in both branches at base-case analysis.

The total monthly average diabetes-related drug cost per patient was calculated taking into account that patients used different types of medications. Information used in this calculation included the percentage of patients taking 1,2 , or 3 types of medications in each group, the average wholesale price, and the medication adherence rate. For example, the average monthly cost of oral diabetic agents $=\%$ of patients on 1 agent $\times$ price of 30 -day supply of metformin $+\%$ of patients on 2 agents of metformin and glipizide $\times$ price of 30-day supply of the 2 agents $+\%$ of patients on the 3 agents of metformin, glipizide, and pioglitazone $\times$ price of 30-day supply of the 3 agents. For other medications, including insulin, antihypertensive medications, and antihyperlipidemic medications, the monthly cost per patient was approximated by the percentage of patients on a type of medication multiplied by the corresponding drug costs (Table 3).

Total wage paid to the pharmacist per patient per year was calculated based on the hourly wage rate and the total number 


\section{A Markov Model of the Cost-Effectiveness of Pharmacist Care for Diabetes in Prevention of Cardiovascular Diseases: Evidence from Kaiser Permanente Northern California}

\section{TABLE 1 Major Clinical Inputs to UKPDS Risk Engine Version 2 and Estimated CVD Risks}

A. Major Clinical Inputs to UKPDS Risk Engine Version 2

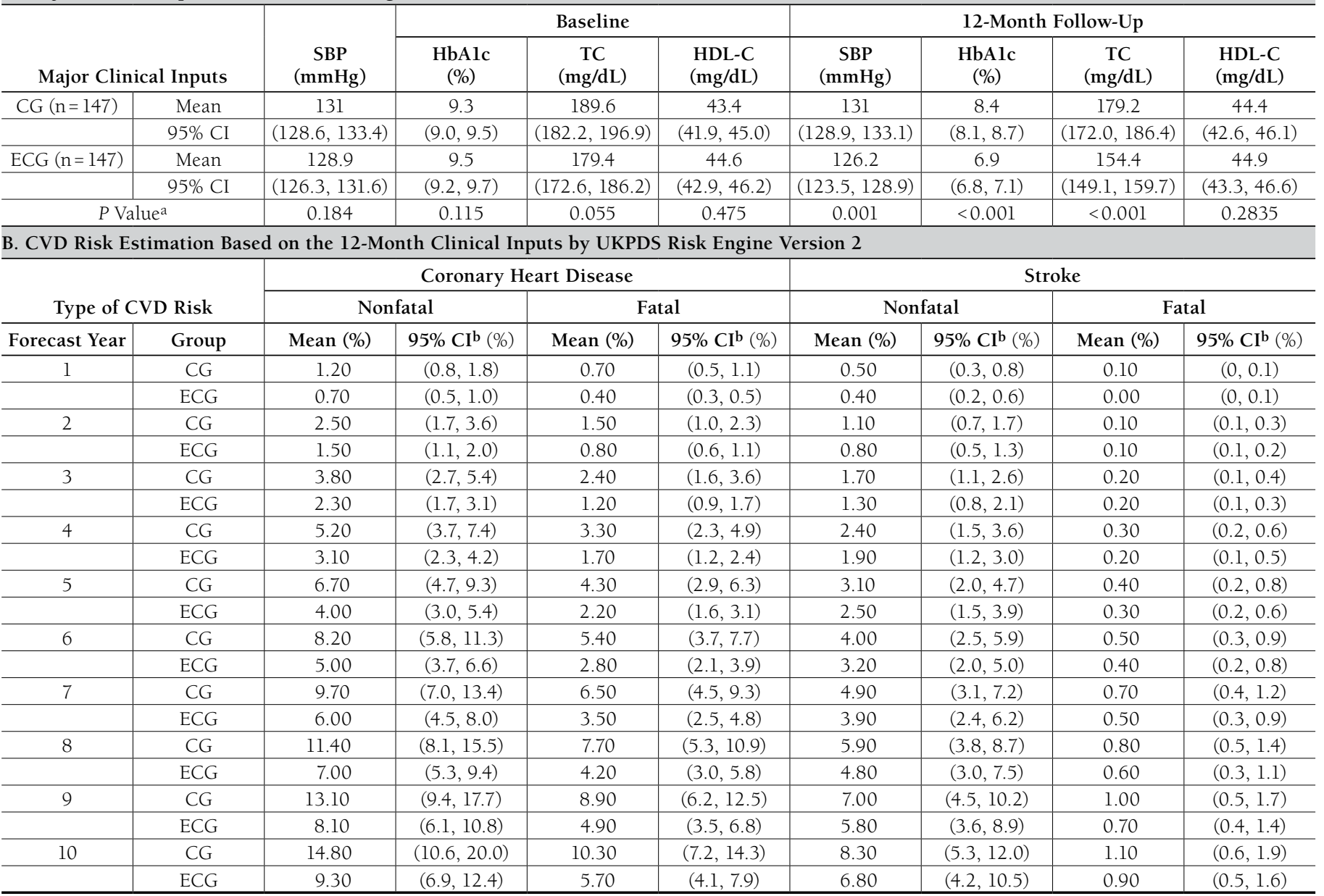

aP values were obtained from nonparametric Wilcoxon signed-ranks tests.

${ }^{b}$ UKPDS Risk Engine (version 2) provided the 95\% CI for each patient for each type of CVD risk and the means of those risks for the 147 patients in each group were reported.

$C G=$ control group; $C I=$ confidence interval; $C V D=$ cardiovascular disease; $E C G=$ enhanced care group; HbAlc=hemoglobin $A 1 c ; H D L-C=$ high-density lipoprotein cholesterol; SBP= systolic blood pressure; TC = total cholesterol; UKPDS = United Kingdom Prospective Diabetes Study.

of hours spent on initial consultation and follow-up phone calls per month. The average length of consultation was estimated to be 45 minutes for the initial consultation (face to face) and 15 minutes for each follow-up visit (typically via telephone) as recorded from the clinical study. The average number of initial consultation and follow-up visits were identified in the enhanced care group as shown in Table 3. The total physician visit fee per year was calculated by the fee per visit multiplied by the average number of visits per year in each group.

\section{Utility Measures}

The utility measures for each of the health states in the Markov model were obtained either from the literature or were based on assumptions due to lack of evidence. Utility weight is the degree of preference individuals have for a particular health state or condition. This weight can vary between 0 and 1 . By definition, a value of 1 represents perfect health and a value of 0 represents death. A discount rate of $5 \%$ was applied to both branches.

All assumptions were subjected to sensitivity analysis (SA). The model assumed that patients have lower utility after the first event of CHD or stroke than patients who remain free of CVD events. In addition, patients will experience a disutility for each additional CHD or stroke after the first event. The utility values used in the study are shown in Table 2. 


\section{A Markov Model of the Cost-Effectiveness of Pharmacist Care for Diabetes in Prevention of Cardiovascular Diseases: Evidence from Kaiser Permanente Northern California}

TABLE 2 Utility Weights for Health

States and Increased Risk of Death Due to CVD Events

\begin{tabular}{l|c|c|c}
\hline & Base Case & $\begin{array}{c}\text { Range for } \\
\text { Sensitivity } \\
\text { Analysis }\end{array}$ & Sources \\
\hline
\end{tabular}

Utilities for each health states in the Markov model

\begin{tabular}{l|c|c|c}
\hline Post-CHD & 0.77 & $\pm 30 \%$ base & Gage et al. ${ }^{40}$ \\
\hline Post-STKa & 0.675 & $(0.6-0.9)$ & Lampe et al. ${ }^{18}$ \\
\hline Additional CHD & -0.055 & $\pm 30 \%$ base & Clarke et al. ${ }^{41}$ \\
\hline Additional STK & -0.164 & $\pm 30 \%$ base & Clarke et al. ${ }^{41}$ \\
\hline Well & 0.782 & $\pm 30 \%$ base & Clarke et al. ${ }^{41}$ \\
\hline Death & 0 & &
\end{tabular}

Relative risk of mortality: increase in risk of mortality relative to all causes mortality

\begin{tabular}{l|l|l|l}
\hline STK & 2.315 & $(1-4.6)$ & Lampe et al. ${ }^{18}$ \\
\hline CHD & 3.716 & $(3-4.7)$ & Dennis et al. ${ }^{19}$ \\
\hline
\end{tabular}

aTo estimate the stroke utility in the main analysis, it was assumed in the study that $70 \%$ of initial strokes were nondisabling, $15 \%$ partially disabling, and $15 \%$ disabling, based on data from the literature.

$C H D=$ coronary heart disease $C V D=$ cardiovascular disease; $S T K=$ stroke.

\section{Model Outputs}

The key outcomes of interest in the model were incremental cost per quality-adjusted life-year (QALY) gained and life-years gained. The total costs per patient calculated for each arm included costs for medications, treatment costs for nonfatal CVD events, labor costs for pharmacists, and physicians visit fee. Life-years were calculated with different time horizons of the model.

\section{Major model assumptions.}

1. The base-case scenario of this Markov model assumed that the cohort of simulating patients in both groups achieved the mean values of clinical outcomes (Alc, SBP, TC, and HDL-C) observed at the end of the 12-month follow-up period.

2. For each set of clinical inputs, UKPDS generated a $95 \%$ confidence interval $(\mathrm{CI})$ around the estimated mean risks. Mean risks based on clinical inputs at the 12-month followup were used in the base-case analysis, while the upper bounds of the $95 \%$ CI of risks was evaluated in the worst case scenario and the lower bounds of the $95 \% \mathrm{CI}$ of the risks in the best case scenario.

3. It was assumed in the base-case analysis that the probabilities of the second and third event would be the same as that of the first-time event predicted by the UKPDS. In recognition of the fact that the UKPDS equation is for a first event only, ${ }^{20}$ the assumption in the base-case analysis was relaxed in the scenario analysis where the mean odds ratio of the second or third event was 2.33 and ranged from 1.67 to 2.79 .

4. To account for the possibility that not all patients will achieve the observed average outcome, we varied the
TABLE 3 Parameters of Cost and Utilization of Medical Services Used in the Model

\begin{tabular}{|c|c|c|c|}
\hline Cost & $\begin{array}{l}\text { Value for Base- } \\
\text { Case Analysis }\end{array}$ & $\begin{array}{l}\text { Range for } \\
\text { Sensitivity } \\
\text { Analysis }\end{array}$ & Source \\
\hline \multicolumn{4}{|l|}{ CVD events } \\
\hline CHD & $\$ 37,462$ & $\pm 50 \%$ base & Straka et al. ${ }^{37}$ \\
\hline Stroke & $\$ 32,916$ & $\pm 50 \%$ base & Straka et al. ${ }^{37}$ \\
\hline \multicolumn{4}{|l|}{ Labor cost } \\
\hline Hourly wage of pharmacist & $\$ 56.20$ & $\$ 40-\$ 80$ & $\begin{array}{c}\text { Bureau of Labor } \\
\text { Statistics }\end{array}$ \\
\hline Physician visit fee & $\$ 60$ & $\$ 50-\$ 100$ & $\begin{array}{c}\text { Kaiser } \\
\text { Permanente }{ }^{39}\end{array}$ \\
\hline \multicolumn{4}{|l|}{ Utilization of medications } \\
\hline $\begin{array}{l}\text { Percentage using insulin } \\
(\mathrm{ECG} / \mathrm{CG})^{\mathrm{a}}\end{array}$ & $0.6 / 0.1$ & $\pm 50 \%$ base & \multirow{3}{*}{ Ip et al. ${ }^{8}$} \\
\hline $\begin{array}{l}\text { Percentage using BP drugs } \\
\text { (ECG/CG) }\end{array}$ & $76.20 / 75.50$ & $\pm 50 \%$ base & \\
\hline $\begin{array}{l}\text { Percentage using } \\
\text { antihyperlipidemic } \\
\text { medications (ECG/CG) }\end{array}$ & $89.80 / 81.60$ & $\pm 50 \%$ base & \\
\hline
\end{tabular}

Average cost of medication per month ${ }^{\mathrm{a}}$

\begin{tabular}{|c|c|c|c|}
\hline Insulin ${ }^{b}$ & $\$ 80.50$ & $\$ 50-100$ & \multirow{3}{*}{$\begin{array}{c}\text { Prices were } \\
\text { obtained from } \\
\text { www.drugs.com }\end{array}$} \\
\hline Lipid and BP medications ${ }^{\mathrm{C}}$ & $\$ 36.50$ & $\$ 25-50$ & \\
\hline $\begin{array}{l}\text { Oral diabetic agents } \\
(E C G / C G)^{d}\end{array}$ & $\$ 52.30 / \$ 44.50$ & $\$ 30-60$ & \\
\hline
\end{tabular}

\section{Utilization of other medical resources}

\begin{tabular}{l|c|c|c}
\hline $\begin{array}{l}\text { Number of pharmacist } \\
\text { face-to-face visits }\end{array}$ & 1.2 & $1.1-1.3$ & \\
\cline { 1 - 2 } $\begin{array}{l}\text { Number of follow-up } \\
\text { phone calls }^{\mathrm{e}}\end{array}$ & 9.5 & $8.4-10.6$ & \multirow{2}{*}{ Ip et al. ${ }^{8}$} \\
\cline { 1 - 2 } Number of physician visits & & \\
\hline $\begin{array}{l}\text { Baseline adherence } \\
\text { rate of drugs }\end{array}$ & 2.3 & $2.1-2.5$ & Murray et al. ${ }^{22}$ \\
\cline { 1 - 2 } $\begin{array}{l}\text { Improvement in } \\
\text { adherence rate }\end{array}$ & $15 \%$ & $10 \%-30 \%$ & Murray et al. ${ }^{22}$ \\
\hline
\end{tabular}

aThis is the price for a 30-day supply adjusted by the proportion of patients using the medications.

${ }^{b}$ The average whole sale price of Novolin N/R was used because this is the drug typically used according to the pharmacists in charge of this study.

'The sum of whole sale price of most prevalent statin and lisinopril was used in the calculation of the average costs adjusted by the percentage of patients on those medications.

dThe price was the average price of 3 types of oral agents (metformin, glipizide, and Actos) weighted by the percentage of patients using 1,2, and 3 different types of agents in the enhanced care group and control group.

'Data were obtained from the study sample as described in the Ip et al. study (2012). ${ }^{8}$

$B P=$ blood pressure $; C G=$ control group; $E C G=$ enhanced care group

parameter indicating the proportion of patients in the enhanced care group who might not respond to the intervention and revert to their baseline Alc, SBP, and lipid levels. The mean 1- to 10-year risks were then recalculated using the baseline values of those clinical markers instead of the 12-month value as the prediction input. Hence, the transition probabilities of CVD risks for the enhanced 


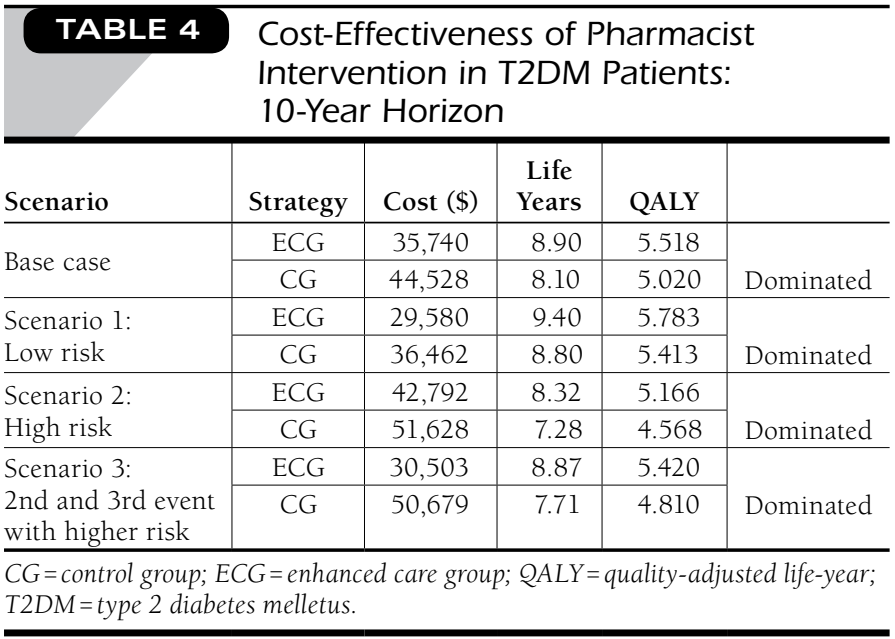

care group were then calculated as a weighted average: the percentage of patients who will maintain the improved outcomes $\times$ CVD risks estimated using the 12-month clinical inputs + (1- the percentage of patients who will maintain the improved outcomes) $\times$ CVD risk estimated using the baseline clinical inputs.

5. It was assumed that the control group had continued to maintain the base line adherence rate of $65 \%$, whereas the enhanced care group had achieved a higher adherence rate and, hence, had higher medication costs (based on expert opinion and other published evidence). ${ }^{22}$

\section{Base-Case Analysis and Scenario Analysis}

In the base-case analysis, the Markov model was estimated using the mean CVD risks for the 10-year follow-up period. The incremental cost-effectiveness ratio (ICER) was calculated, and the analysis was then repeated for 2 other scenarios where the lower bound and the upper bound of 95\% CI were used. TreeAge $\mathrm{Pro}^{23}$ was used to perform decision analyses. A third scenario analysis was conducted assuming the second or third CVD event was 2.33 times more likely to occur than estimated by the UKPDS Risk Engine.

\section{Sensitivity Analysis}

SA was conducted to test the robustness of the baseline results. Three types of SA were performed: one-way SA, multiple oneway SA, and probabilistic SA. All the variables were examined using the plausible range specified in tables 1 and 2 for the deterministic SA. Finally, Monte Carlo simulation was conducted both for the base-case and third scenario to allow all variables to vary simultaneously in a further effort to assess the robustness of our findings. For this analysis, variables related to costs were assigned log-normal distributions; number of visits and follow-up phone calls were assigned Poisson distribution; while all others were given uniform distribution within the assumed plausible range.
This study was approved by both Touro UniversityCalifornia Institutional Review Board (IRB Application no. P-0909) and KP Northern California Institutional Review Board (IRB Application no. CN-09EIp-01-H).

\section{Results}

\section{Base-Case and Scenario Analyses}

Table 4 presents the results of the economic evaluation of the enhanced care group relative to the control group. The model suggests that the enhanced control group dominated the control group with lower treatment cost $(\$ 35,740$ vs. $\$ 44,528)$ per patient and more life years (8.9 vs. 8.1) and QALY (5.51 vs. 5.02) over the 10 -year period. Three types of scenario analysis were conducted. The dominant results of the enhanced care group remained even after evaluating the worst case scenario, when the upper bound of the $95 \% \mathrm{CI}$ of the risks was used, and the best-case scenario, when the lower bounds of the 95\% CI of the risks were applied. In the third scenario, the transition probabilities for the first-time CVD event remained the same as the base case, but the risks for the second and third CVD events were set to 2.33 times higher than the estimated firsttime probability by UKPDS. ${ }^{20}$ Again, the dominant results of the enhanced care group were repeated.

\section{Sensitivity Analysis}

Multiple one-way SA. Appendix A shows a selection of the one-way SA (tornado diagram) for the net monetary benefit of using enhanced care. In this diagram, each bar represents the impact of uncertainty in an individual variable on the results. The horizontal bar was generated for each selected variable when the baseline estimate of the variable was varied over plausible ranges (tables 2 and 3), with a wider bar indicating a greater potential effect on the monetary benefit. All parameters were varied around the base-case value within a certain range as specified. The variable identified in the tornado diagram as having the largest impact on the net monetary benefit given a threshold willingness to pay at \$50,000/QALY was the time horizon of this analysis, followed by the utility of the health status for diabetic patients free of CVD events (Appendix A). However, all variations in net monetary benefits due to these variables were within the positive range, demonstrating that the enhanced care group remained the preferred strategy. All the remaining variables had nearly no impact on the net monetary benefits.

One-way SA. One-way SA was conducted to examine the individual impact of the adoption of different time horizons on the study outcomes. It is worth noting that as the time horizon was extended for the analysis, the net monetary benefits of the enhanced care group versus the control group increased (see Appendix B). It seems that a minimum of 4 years is needed for the intervention program to achieve higher net monetary 


\section{A Markov Model of the Cost-Effectiveness of Pharmacist Care for Diabetes in Prevention of Cardiovascular Diseases: Evidence from Kaiser Permanente Northern California}

\section{FIGURE 2 Cost-Effectiveness Acceptability by Time Horizon of the Model}

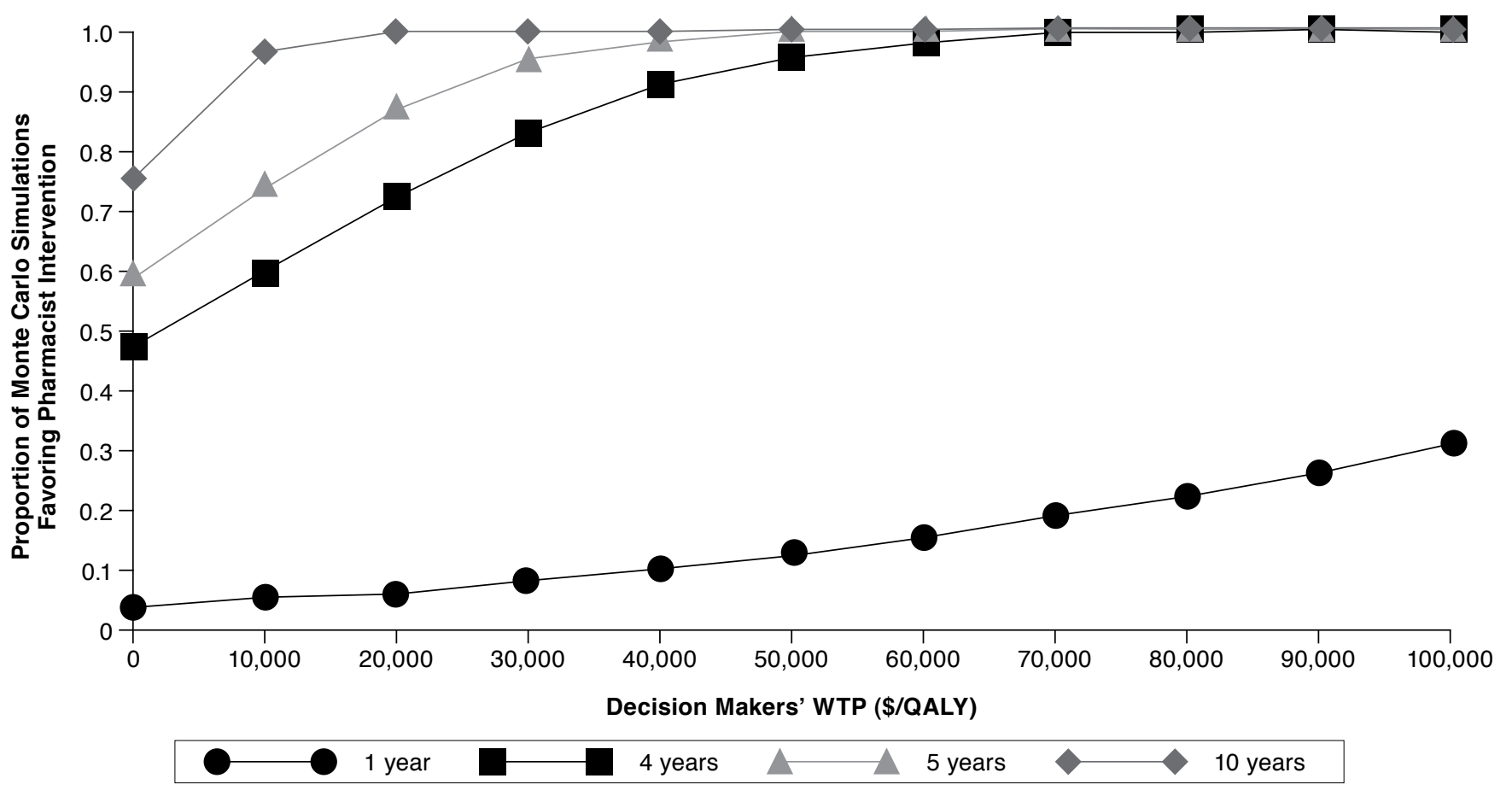

$C E=$ cost-effectiveness; $Q A L Y=$ quality adjusted life years; $W T P=$ willing to pay.

benefits than the control group while holding all other parameters at the base-case scenario. However, this threshold was shortened to 3.3 years when the scenario was changed to the situation that the second and third event had higher odds $(\mathrm{OR}=2.33)$ than the first event.

Probabilistic sensitivity analysis results. A cost-effectiveness acceptability curve (Figure 2) showed the likelihood of the intervention being considered cost-effective compared with the control care at various levels of willingness to pay by the payers. Enhanced care was shown to have a consistently higher chance of being the favored strategy regardless of the level of willingness to pay (WTP) if a longer time horizon was adopted: 10 years for base case scenario (Figure 2) and 7 years for the third scenario. In both situations, the shorter the time horizon adopted, the lesser the chance for enhanced care to be preferred. If a 1- to 3-year time horizon was adopted, adding pharmacists into the health care team became less likely to be preferred than using a PCP only in both cases. It seems that at the level of WTP equal to \$50,000/QALY, a 5-year time horizon in the base case is the minimum length of time for the program to become the favored strategy given the uncertainty of other factors. However, the minimum length was shortened to 4 years in the third scenario analysis, when second or third events were presumed to occur at a higher rate with OR of 2.33 .
Therefore, probabilistic SA results reinforced the importance of correlation between the length of intervention and the magnitude of preventive effects in CVD risk reduction in determining the likelihood of cost-effectiveness of enhanced care.

\section{Discussion}

Our study, a comprehensive cost-effectiveness analysis of a pharmacist-led diabetes management intervention in a primary care setting, shows that pharmacist intervention can help reduce the long-term CVD risk among patients with T2DM while reducing costs and increasing QALYs. Taking into account the long-term cost savings associated with fewer CVD events, enhanced care (which involves the pharmacist as a member of the primary care team) is a dominant strategy (cost and life saving) compared with the control group (PCP only). The findings of the study were quite robust. The results suggested that it would be cost-effective to incorporate pharmacists into the health care management team for diabetic patients.

It is worth mentioning that previous literature has also documented the effectiveness of a variety of interventions by other health care professionals as well. Diabetes management interventions delivered individually or as a team by physicians, nurses, pharmacists, and other diabetes educators 


\section{A Markov Model of the Cost-Effectiveness of Pharmacist Care for Diabetes in Prevention of Cardiovascular Diseases: Evidence from Kaiser Permanente Northern California}

have been shown to improve patient outcomes in numerous studies. ${ }^{24}$ However, our study differs from previous studies in the intervention strategy, sample populations, and the outcomes and time horizons examined. In order for decision makers to gauge the potential of cost-effectiveness of our pharmacist-led intervention relative to other types of intervention, it is necessary to compare the studies that evaluated other types of intervention with ours. A recent cost-effectiveness analysis study found that nurse specialists gave diabetes care according to a pre-set protocol that was similar to care provided by physicians in terms of quality of life and economic value but with potential savings due to lower labor costs. ${ }^{25}$ In a recent meta-analysis of 11 predefined quality improvement (QI) strategies for diabetes, it was shown that the most effective QI strategy was team changes (i.e., the pharmacist or nurse has an active role in patient monitoring and adjusting drug regimens) with further Alc reduction of $0.33 \%$ (95\% CI $0.28-0.45 ; 120$ trials), LDL cholesterol by 0.10 millimoles per liter (mmol/L; 0.05-0.14; 47 trials), SBP by 3.13 (2.19-4.06; 65 trials), and diastolic blood pressure (DBP) by $1.55 \mathrm{mmHg}\left(0.95-2.15 ; 61 \mathrm{mmHg}\right.$ trials) versus usual care. ${ }^{26}$ Of particular importance, QI strategies that allowed pharmacists and specialist nurses to make independent changes to drug therapies were found to be most effective. This is exactly the major feature of our pharmacist-led intervention program. Our intervention program has achieved a mean Alc reduction from $9.5 \%$ to $6.9 \%$ compared with the reduction from $9.3 \%$ to $8.4 \%$ in the control group after 12 months, resulting in a $1.7 \%$ improvement $(P<0.001)$. The patients under enhanced care also experienced a mean reduction of LDL by $10.1 \mathrm{mg} /$ $\mathrm{dL}$, a reduction of SBP by $2.6 \mathrm{mmHg}$, and a reduction of DBP by $2.3 \mathrm{mmHg}$. According to the UKPDS, a 1\% decrease in Alc is associated with a $37 \%$ reduction in microvascular complications and a $21 \%$ reduction in the risk of any diabetes-related complication or death. ${ }^{19}$ The degree of Alc reduction due to enhanced care would potentially translate into a 96\% decrease in microvascular complications and a 55\% reduction in any diabetes-related complication or death. ${ }^{26}$ Using the UKPDS Risk Engine, we found that a 2.6\% reduction in Alc among the enhanced care group reduced their 10-year nonfatal CHD risk from $16.4 \%$ to $9.3 \%$ (a $43 \%$ reduction ) and the fatal CHD risk from $11.3 \%$ to $5.7 \%$ (a $50 \%$ reduction). ${ }^{8}$

In terms of the findings about costs, available data from the Asheville Project, a longitudinal pre/post cohort study, showed that diabetes patients receiving care from a community pharmacist reduced their mean total direct medical costs by $\$ 1,200$ while maintaining clinically meaningful improvements in their Alc over a 5-year follow-up period. ${ }^{9}$ The Diabetes Ten City Challenge, a multisite community pharmacy health management program for diabetes patients, also showed a \$1,079 in average total health care costs per patient per year. ${ }^{10}$ In our study, we found that the average annual costs over the 10-year period was $\$ 3,574$ in the enhanced care group versus $\$ 4,453$ in the care group at base case (Table 4), which translates to $\$ 879$ savings per year. Another economic study evaluating the cost-effectiveness of strategies for managing people at high risk for diabetes found that the annual diabetes-related cost of an average patient with diabetes was $\$ 4,121 .{ }^{27}$ Therefore, the costs incurred by our intervention and cost savings due to the intervention are comparable to other interventions as well. In terms of QALY, the study focusing on life-style intervention among diabetic patients found that the QALY in the 30-year period was calculated as $11.478^{27}$ with an average of 0.38 QALY per year. Another study conducted in Europe ${ }^{28}$ found that intensive treatment among diabetic patients with 18.6 years of survival had a QALY of 10.2, with an average of 0.5 QALY per year, and it offered a QALY gain of 0.094 per year compared with conventional therapy. In contrast, our study of the 10-year outcome found that the QALY per year averages 0.45-0.57 QALY (Table 4), offering a QALY gain of 0.04-0.07 per year. The higher QALY per year compared with the life-style study might be due to the difference in the cohorts of patients in the 2 studies and the model horizon. The previous study included patients with higher risk for diabetes in the model, and they tended to be older and thus have lower quality of life. However, the lower QALY per year compared with the European study might be because the utility weight assigned to diabetic patients is 0.814 as opposed to 0.782 in our study.

Notably, the SA suggested that 1 of the driving factors of the cost-effectiveness of this program is the time horizon adopted by decision makers. This is partly because the longer-term CVD risk reduction was more dramatic than the short-term reduction as estimated by the UKPDS Risk Engine. Based on our research, if health plans were willing to pay $\$ 50,000 /$ QALY, it would take at least 4-5 years for the addition of a clinical pharmacist to the care team to ensure cost-effectiveness to cover all uncertainties factored into the model.

One of the strengths of our study is that the model was primarily based on real-world data, which included clinical effectiveness and health care utilization data collected from 2 comparable clinics with matching baseline data to reduce bias. This is an improvement compared with previous studies that lacked comparison groups and had short follow-up periods (less than 1 year), smaller sample sizes, unequal baseline characteristics across groups, and lack of cardiovascular markers such as BP and lipid levels. ${ }^{3}$ Also, a Markov model was used to simulate 2 matched hypothetical cohorts of patients to estimate the long-term CVD risk reduction.

Another unique contribution of our modeling study was the use of the UKPDS Risk Engine in the projection of cardiovascular outcomes over the long term based on short-term clinical surrogates for T2DM patients. The Risk Engine was 


\section{A Markov Model of the Cost-Effectiveness of Pharmacist Care for Diabetes in Prevention of Cardiovascular Diseases: Evidence from Kaiser Permanente Northern California}

derived from UKPDS data and was designed to reflect risk in the general T2DM population; therefore, it provided more relevant predictions than the Framingham score adopted by a recent publication. ${ }^{29}$ The reliability of using the Framingham equation to calculate CHD risk in diabetes patients has been questioned because of the small proportion of diabetes patients in the Framingham study. ${ }^{30}$ For example, Ladhani et al. (2012) utilized the UKPDS Risk Engine, which is considered a more reliable method of measuring cardiovascular risk in T2DM patients. ${ }^{31}$ One of the concerns for those adopting the intervention program in the long term might be the difficulty in maintaining the improved outcomes observed at the l-year follow-up. This concern was addressed in our study by varying the ranges of the key variables-the percentage of patients who would be able to maintain the achieved outcomes from $50 \%$ to $100 \%$ in the SA. Notably, this variable had minimum impact on the net monetary benefit, suggesting that the approach would still be cost-effective even if a proportion of patients would not be able to maintain the identified improvement as shown in this study.

As the prevalence of diabetes continues to increase and changes in the health care system because of the Patient Protection Affordable Care Act take effect, millions of new patients are expected to need care. The ongoing shortage of primary care practitioners, coupled with the increasing numbers of patients with T2DM, pose significant challenges to the health care system. ${ }^{32}$ In the face of this shortage, several health care professionals have stepped in to fulfill this role. A recent meta-analysis showed that team-based interventions (i.e., pharmacist or nurse involved in patient monitoring and adjusting drug regimens) was the most effective diabetes management strategy compared with several others. ${ }^{26}$ In a recent report to the U.S. Surgeon General, pharmacists were singled out as health care professionals who manage disease through medications and other patient care services but are not recognized as health care providers by national health policy in spite of evidence to the contrary. ${ }^{32}$ Given their expertise and experience, coupled with their accessibility, pharmacists are uniquely positioned to play a much larger role in the health care delivery system.

Currently, the accountable care organization (ACO) concept is being promoted as an integrated approach to managing chronic conditions such as diabetes and is partly driven by the Affordable Care Act. ${ }^{33}$ Although the act was not explicit about including pharmacists as part of the health care team, ${ }^{33}$ the U.S. Surgeon General recently recognized that "pharmacy practice models (implemented in collaboration with physicians or as part of a health team) improve patient and health system outcomes and optimize primary care access and delivery." 32 This study provides further evidence of the beneficial clinical and economic potential of including pharmacists in ACOs. In addition, this study also brings attention to the finding that the length of the enrollment period determines the economic benefits of the pharmacists' preventive efforts. It emphasizes the notion that payers might not be able to reap the benefits of preventive intervention programs targeted at patients with chronic diseases unless they can keep the patients in their plans for a certain period of time.

\section{Limitations}

There are limitations to this study worthy of discussion. First, this study relies heavily on evidence generated from a retrospective cohort analysis and was performed at 2 medical facilities using a quasi-experimental study design. A randomized controlled study including a greater number of patients, involving more pharmacist providers and incorporating multiple medical facilities would further enhance the quality of the clinical and economic evidence.

Second, there were different aspects to the pharmacist intervention in this study. However, the quasi-experimental study design did not allow us to estimate the individual effects of those actions on the costs and benefits. Specifically, the improved outcomes in the pharmacist intervention group may have been because of changes in drug dosing, changes of drug regimen, the face-to-face clinical consultation, frequent followup phone visits, or improved medication adherence. Those actions were not compared between groups directly; therefore, it is impossible to disentangle the possible multidimensional effects of various intervention strategies. Consequently, it may be difficult for decision makers to decide on the focus of the intervention. Ideally, if it could be established that the frequent pharmacist follow-up phone visits helped improve medication optimization and adherence rates, then the provider might be interested in investing in an intervention program with these features. ${ }^{34,35}$ If it were the face-to-face contact with pharmacists that was more effective, then the provider might need to make sure adequate physical space (i.e., exam room or private office) is available in the clinical setting. However, the study was not able to identify which management scheme would help contribute most to the improved outcomes. Future studies are necessary to examine the direct impact of specific management strategies on the cost-effectiveness of various pharmacist intervention programs.

A related concern is that the patients in the control group in the retrospective study also experienced various degrees of improvement in outcomes at the end of 12 months. ${ }^{8}$ Similarly, there is no way to identify the specific causes in the control group responsible for the improvement. If actions taken by pharmacists in the enhanced care group also occurred in the control group, such as monitoring and correction of inappropriate medication use, this could lead to biased estimates of the impact of the intervention. However, this would bias the 


\section{A Markov Model of the Cost-Effectiveness of Pharmacist Care for Diabetes in Prevention of Cardiovascular Diseases: Evidence from Kaiser Permanente Northern California}

results of the study against the intervention group. Another related concern is that our implication for health plans as to the length of intervention required for the same cohort of enrollees in their plans is not clear-cut. Although 4-5 years are identified to be the preferred time horizon, the choice also depends on the magnitude of CVD risk reduction. Four years is found to be the minimum length when second or third CVD events have higher odds to occur, while a longer period of 5 years is needed when assuming all CVD events occur at the probability of a first-time event.

Finally, pharmacist salaries have been a source of concern for providers when considering the implementation of this sort of program. ${ }^{11}$ This study suggested that the pharmacist costs have no significant impact on the cost efficiency of implementing the program in the KP HMO setting. There are a few reasons why we should be careful in generalizing the results to other clinical settings. For example, other health care delivery systems might differ from KP in major areas such as facility infrastructure, logistical planning, upfront training costs of pharmacists qualified for managing medication therapy, the nature of the intervention, and the patient population-all of which might influence the cost calculation algorithm. In addition, the KP diabetes population is drawn from an insured population in northern California and may not be representative of other geographic regions, the United States, or other health plans. ${ }^{36}$

\section{Conclusions}

In this analysis, we estimated that adding pharmacists to the health care team for the direct management of diabetic patients significantly improved long-term CVD risks. The estimated longer-term CVD risk reduction appears more dramatic than the short-term reduction. The results of the economic model suggest that whether pharmacist intervention provides a cost-effective management tool crucially depends on the length of the effective period of intervention, which is largely determined by the length of time that the patient is enrolled in the health plan. Considering all sources of uncertainty, it seems that a minimum of 4-5 years of consistent enrollment is required for the intervention to be cost-effective without much uncertainty. The study provides insights that will be beneficial for payers to determine whether it is feasible to add pharmacists to the health care team for the direct management of diabetes patients. Future research is needed to improve knowledge about the relative cost-effectiveness of the specific interventions performed by pharmacists in different clinical settings.

\section{Authors}

JUNHUA YU, PhD, MS, is Assistant Professor, Department of Social, Behavioral, and Administrative Science; BIJAL M. SHAH, $\mathrm{PhD}$, is Associate Professor, Department of Social, Behavioral, and Administrative Science; and ERIC J. IP, PharmD, BCPS, CSCS, CDE, is Associate Professor, Department of Pharmacy Practice, Touro University College of Pharmacy, Vallejo, California. ERIC J. IP, PharmD, BCPS, CSCS, CDE, is also Diabetes Clinical Pharmacist, Department of Internal Medicine, Kaiser Permanente Mountain View Clinics, Mountain View, California. JAMES CHAN, PharmD, PhD, is Pharmacy Quality and Outcomes Coordinator, Kaiser Permanente Medical Care Program, Pharmacy Outcomes Research Group, Oakland, California.

AUTHOR CORRESPONDENCE: Junhua Yu, PhD, MS, Assistant Professor, Department of Social, Behavorial, and Administrative Science, Touro University College of Pharmacy, 1310 Club Dr., Mare Island, Vallejo, CA 94592. Tel.: 707.638.5913; E-mail: junhua.yu@tu.edu.

\section{DISCLOSURES}

This research was funded by the American Association of Colleges of Pharmacy (AACP) New Pharmacy Faculty Research Awards program.

Concept and design were performed by $\mathrm{Yu}$, Shah, Ip, and Chan. Data were collected by Yu, Ip, and Shah and interpreted by Ip, Chan, Yu, and Shah. Yu, Shah, Ip, and Chan wrote and revised the manuscript.

\section{REFERENCES}

1. University of Pittsburgh Diabetes Prevention Support Center. Diabetes $\&$ related conditions_cardiovascular disease. Available at: http://www. diabetesprevention.pitt.edu/cardiovasculardisease.aspx. Accessed February 26, 2013.

2. Pasco JA, Jacka FN, Williams LJ, et al. Clinical implications of the cytokine hypothesis of depression: the association between use of statins and aspirin and the risk of major depression. Psychother Psychosom. 2010;79(5):323-25.

3. Wubben DP, Vivian EM. Effects of pharmacist outpatient interventions on adults with diabetes mellitus: a systematic review. Pharmacotherapy. 2008;28(4):421-36.

4. Koenigsfeld CF, Horning KK, Logemann CD, Schmidt GA. Medication therapy management in the primary care setting: a pharmacist-based payfor-performance project. J Pharm Pract. 2012;25(1):89-95

5. Padiyara RS, D'Souza JJ, Rihani RS. Clinical pharmacist intervention and the proportion of diabetes patients attaining prevention objectives in a multispecialty medical group. J Manag Care Pharm. 2011;17(6):456-62.

6. Kraemer DF, Kradjan WA, Bianco TM, Low JA. A randomized study to assess the impact of pharmacist counseling of employer-based health plan beneficiaries with diabetes: the EMPOWER study. J Pharm Pract. 2012;25(2):169-79.

7. Clarke PM, Gray AM, Briggs A, et al. A model to estimate the lifetime health outcomes of patients with type 2 diabetes: the United Kingdom Prospective Diabetes Study (UKPDS) Outcomes Model (UKPDS no. 68) Diabetologia. 2004:47(10):1747-59. 


\section{A Markov Model of the Cost-Effectiveness of Pharmacist Care for Diabetes in Prevention of Cardiovascular Diseases: Evidence from Kaiser Permanente Northern California}

8. Ip EJ, Shah BM, Yu J, Chan J, Nguyen LT, Bhatt DC. Enhancing diabetes care by adding a pharmacist to the primary care team: an analysis of shortterm clinical markers and long-term cardiovascular outcomes. Am J HealthSyst Pharm. [in press].

9. Cranor CW, Bunting BA, Christensen DB. The Asheville Project: longterm clinical and economic outcomes of a community pharmacy diabetes care program. J Am Pharm Assoc (Wash). 2003;43(2):173-84.

10. Fera T, Bluml BM, Ellis WM. Diabetes Ten City Challenge: final economic and clinical results. J Am Pharm Assoc (2003). 2009;49(3):383-91.

11. Rothman RL, So SA, Shin J, et al. Labor characteristics and program costs of a successful diabetes disease management program. Am J Manag Care. 2006;12(5):277-83.

12. Taylor SJ, Milanova T, Hourihan F, et al. A cost-effectiveness analysis of a community pharmacist-initiated disease state management service for type 2 diabetes mellitus. Int J Pharm Pract. 2005;13(1):33-40.

13. Jacobs-van der Bruggen MA, van Baal PH, Hoogenveen RT, et al. Costeffectiveness of lifestyle modification in diabetic patients. Diabetes Care. 2009;32(8):1453-58

14. Ramsey SD, Clarke LD, Roberts CS, Sullivan SD, Johnson SJ, Liu LZ. An economic evaluation of atorvastatin for primary prevention of cardiovascular events in type 2 diabetes. Pharmacoeconomics. 2008;26(4):329-39.

15. Stevens RJ, Kothari V, Adler AI, Stratton IM. The UKPDS risk engine: a model for the risk of coronary heart disease in Type II diabetes (UKPDS 56). Clin Sci (Lond). 2001;101(6):671-79.

16. Kothari V, Stevens RJ, Adler AI, et al. UKPDS 60: risk of stroke in type 2 diabetes estimated by the UK Prospective Diabetes Study risk engine. Stroke. 2002;33(7):1776-81.

17. Arias E. United States life tables, 2006. Natl Vital Stat Rep. 2010;58(21):1-40.

18. Lampe FC, Whincup PH, Wannamethee SG, Shaper AG, Walker M, Ebrahim S. The natural history of prevalent ischaemic heart disease in middle-aged men. Eur Heart J. 2000;21(13):1052-62.

19. Dennis MS, Burn JP, Sandercock PA, Bamford JM, Wade DT, Warlow CP. Long-term survival after first-ever stroke: the Oxfordshire Community Stroke Project. Stroke. 1993;24(6):796-800.

20. Prescott A, Bailey JE, Kelly KJ, Munyombwe T, Gray A, Summers LK. The effectiveness and cost of single and multi-factorial cardiovascular risk factor modification to guideline targets in type 2 diabetes. Prim Care Diabetes. 2012;6(1):67-73.

21. Haffner SM, Lehto S, Ronnemaa T, Pyorala K, Laakso M. Mortality from coronary heart disease in subjects with type 2 diabetes and in nondiabetic subjects with and without prior myocardial infarction. N Engl J Med. 1998;339(4):229-34.

22. Murray MD, Young J, Hoke S, et al. Pharmacist intervention to improve medication adherence in heart failure: a randomized trial. Ann Intern Med. 2007;146(10):714-25.

23. TreeAge Pro. TreeAge Software, Inc. Williamstown, MA.

24. Renders CM, Valk GD, Griffin SJ, Wagner EH, Eijk Van JT, Assendelft WJ. Interventions to improve the management of diabetes in primary care, outpatient, and community settings: a systematic review. Diabetes Care. 2001;24(10):1821-33.

25. Arts EE, Landewe-Cleuren SA, Schaper NC, Vrijhoef HJ. The cost-effectiveness of substituting physicians with diabetes nurse specialists: a randomized controlled trial with 2-year follow-up. J Adv Nurs. 2012;68(6):1224-34.
26. Tricco AC, Ivers NM, Grimshaw JM, et al. Effectiveness of quality improvement strategies on the management of diabetes: a systematic review and meta-analysis. Lancet. 2012;379(9833):2252-61.

27. Eddy DM, Schlessinger L, Kahn R. Clinical outcomes and cost-effectiveness of strategies for managing people at high risk for diabetes. Ann Intern Med. 2005;143(4):251-64.

28. Gaede P, Valentine WJ, Palmer AJ, et al. Cost-effectiveness of intensified versus conventional multifactorial intervention in type 2 diabetes: results and projections from the Steno-2 study. Diabetes Care. 2008;31(8):1510-15.

29. Al Mazroui NR, Kamal MM, Ghabash NM, Yacout TA, Kole PL, McElnay JC. Influence of pharmaceutical care on health outcomes in patients with type 2 diabetes mellitus. Br J Clin Pharmacol. 2009;67(5):547-57.

30. Coleman RL, Stevens RJ, Retnakaran R, Holman RR. Framingham, SCORE, and DECODE risk equations do not provide reliable cardiovascular risk estimates in type 2 diabetes. Diabetes Care. 2007;30(5):1292-93.

31. Ladhani NN, Majumdar SR, Johnson JA, et al. Adding pharmacists to primary care teams reduces predicted long-term risk of cardiovascular events in type 2 diabetic patients without established cardiovascular disease: results from a randomized trial. Diabet Med. 2012;29(11):1433-39.

32. Giberson S, Yoder S, Lee MP. Improving patient and health system outcomes through advanced pharmacy practice: a report to the U.S. Surgeon General 2011. Office of the Chief Pharmacist. U.S. Public Health Service. December 2011. Available at: http://www.accp.com/docs/positions/misc/ Improving_Patient_and_Health_System_Outcomes.pdf. Accessed February 19, 2013.

33. Lipton HL. Pharmacists and health reform: go for it! Pharmacotherapy. 2010;30(10):967-72

34. Piette JD, Weinberger M, McPhee SJ, Mah CA, Kraemer FB, Crapo LM. Do automated calls with nurse follow-up improve self-care and glycemic control among vulnerable patients with diabetes? Am J Med. 2000;108(1):20-27.

35. Piette JD, McPhee SJ, Weinberger M, Mah CA, Kraemer FB. Use of automated telephone disease management calls in an ethnically diverse sample of low-income patients with diabetes. Diabetes Care. 1999;22(8):1302-09.

36. Karter AJ, Schillinger D, Adams AS, et al. Elevated rates of diabetes in Pacific Islanders and Asian subgroups: The Diabetes Study of Northern California (DISTANCE). Diabetes Care. 2012. Oct 24. [Epub ahead of print]

37. Straka RJ, Mamdani M, Damen J, et al. Economic impacts attributable to the early clinical benefit of atorvastatin therapy-a US managed care perspective. Curr Med Res Opin. 2007;23(7):1517-29.

38. U.S. Department of Labor. Bureau of Labor Statistics. Occupational employment statistics. Alphabetical list of SOC occupations. Available at: http://www.bls.gov/oes/2002/oes_alph.htm\#M. Accessed February 14, 2013.

39. Kaiser Permanente. 2011 sample fee list for Northern California. January 2011. Available at: http://www.personnel.saccounty.net/Benefits/ Documents/2011SampleFeeList-Ncal.pdf. Accessed February 26, 2013.

40. Gage BF, Cardinalli AB, Albers GW, Owens DK. Cost-effectiveness of warfarin and aspirin for prophylaxis of stroke in patients with nonvalvular atrial fibrillation. JAMA. 1995;274(23):1839-45.

41. Clarke P, Gray A, Holman R. Estimating utility values for health states of type 2 diabetic patients using the EQ-5D (UKPDS 62). Med Decis Making. 2002;22(4):340-49. 


\section{A Markov Model of the Cost-Effectiveness of Pharmacist Care for Diabetes in Prevention of Cardiovascular Diseases: Evidence from Kaiser Permanente Northern California}

\section{APPENDIX A Tornado Diagram for Multiple One-Way Sensitivity Analysis}

\section{Effects on Net Monetary Benefit at Willingness to Pay $=\$ 50,000 / Q A L Y$}

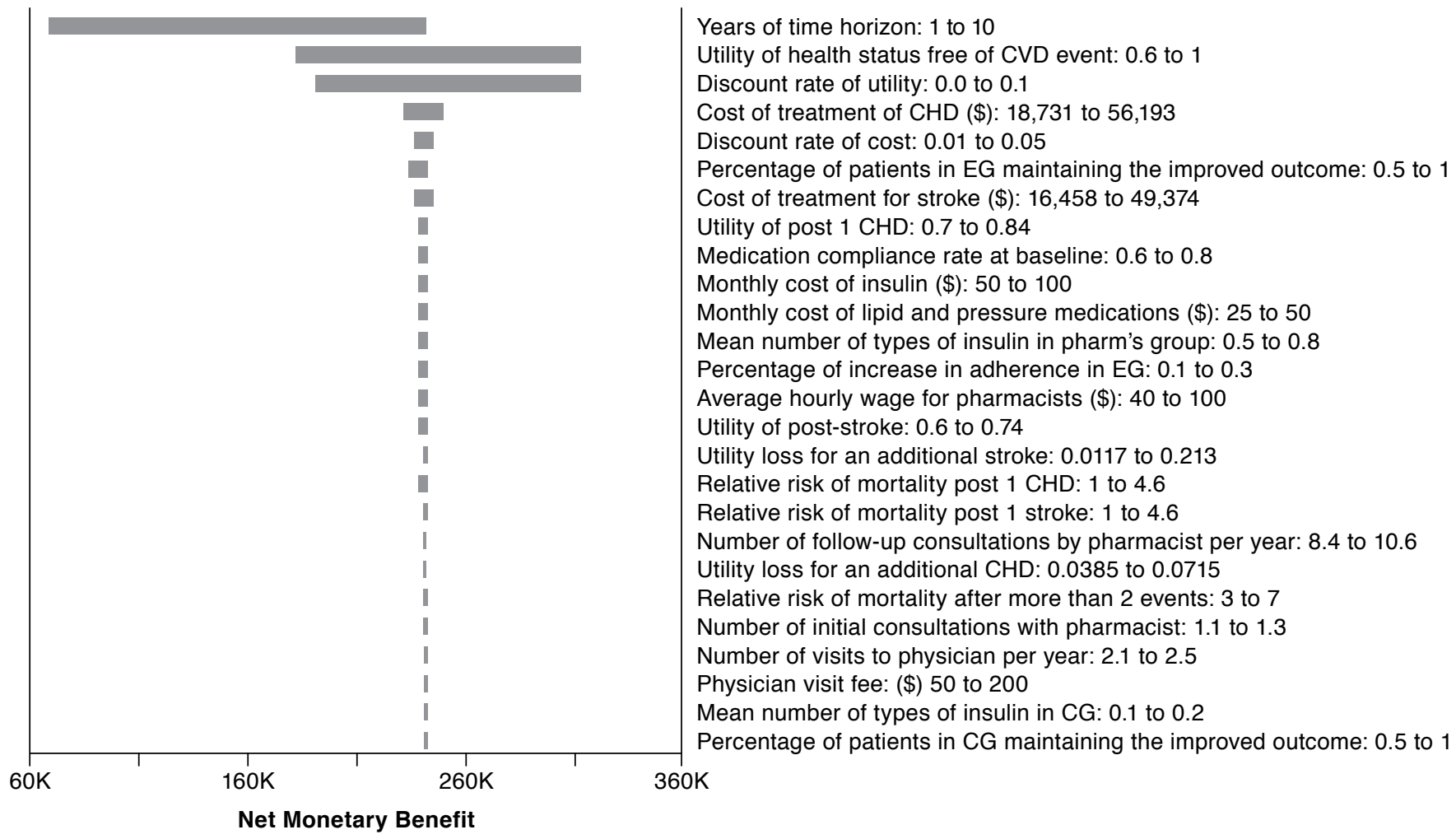

$C G=$ control group $; C H D=$ coronary heart disease $; C V D=$ cardiovascular disease $; E G=$ enhanced care group; $K=$ thousands

\section{APPENDIX B One-Way Sensitivity Analysis}

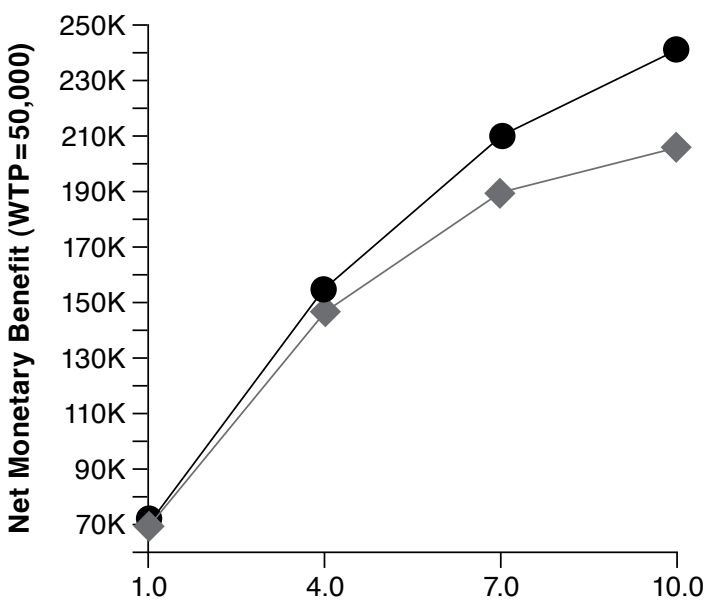

Number of Years Evaluated in Markov Model

Enhanced Care Group Control Group

